\title{
Colaboración y uso de las TIC como factores del desarrollo profesional docente en el contexto educativo uruguayo. Protocolo de análisis para un estudio de casos múltiple
}

\author{
Collaboration and use of ICT as factors of professional teacher \\ development in the Uruguayan educational context. Analysis protocol \\ for a multiple case-study
}

ISSN 1510-2432 - ISSN 1688-9304 (en línea) - DOI: https://doi.org/10.18861/cied.2018.9.1.2818

Mariela Questa-Torterolo

Doctora (Cand.) en Educación, Universitat Autònoma de Barcelona, España. Master en Gestión Educativa, Universidad ORT Uruguay. Licenciada en Relaciones Internacionales. Docente e investigadora en gestión educativa, inclusión de estudiantes en educación superior, formación de formadores y desarrollo profesional. Docente, Instituto de Educación, Universidad ORT Uruguay.

David Rodríguez-Gómez

Doctor en Calidad y Procesos de Innovación Educativa y Licenciado en Pedagogía, Universidad Autónoma de Barcelona, España. Profesor, Departamento de Pedagogía Aplicada e investigador, Equipo de Desarrollo Organizativo,Universidad Autónoma de Barcelona. Profesor colaborador de postgrados, Universitat Oberta de Catalunya e Instituto de Educación, Universidad ORT Uruguay.

Julio Meneses

Doctor en Sociedad de la Información y el Conocimiento, Universitat Oberta de Catalunya, España. Licenciado en Psicología, Universidad de Oviedo, España. Profesor de Metodología de la Investigación en los Estudios de Psicología y Ciencias de la Educación e Investigador en desigualdad digital, incorporación de tecnología a los sistemas educativos y abandono de los estudiantes universitarios, Internet Interdisciplinary Institute (IN3), Universitat Oberta de Catalunya.

Fecha de recibido: 10/10/2017

Fecha de aceptado: 27/03/2018

\section{Resumen}

La colaboración entre docentes, con o sin uso de las tecnologías de la información y la comunicación (TIC), constituye un factor determinante en su desarrollo profesional y, en consecuencia, un elemento clave para el buen funcionamiento de los centros educativos y la calidad del sistema escolar. Por este motivo, y dada la escasez de investigaciones sobre el tema en Uruguay fueron exploradas las formas que adopta la colaboración con fines profesionales y los usos de las TIC con estos fines. El diseño del estudio siguió la estrategia de estudio de caso múltiple para el que se seleccionaron cuatro escuelas primarias y cuatro centros de enseñanza secundaria en el departamento de Colonia. En cada centro se aplicó una entrevista en profundidad al director y a dos docentes identificados por sus actitudes hacia la colaboración a partir de un grupo de discusión realizado entre docentes. Asimismo se recolectaron datos del contexto y se realizaron observaciones no participantes de la infraestructura y del ambiente escolar. Los datos fueron procesados según un protocolo de análisis por capas mediante el que se describieron dos casos en detalle. Luego se buscaron patrones entre dichos casos y temas transversales. Por último se organizaron 
los hallazgos en una meta-matriz para mostrar los vínculos entre qué se identifica como colaboración docente, cuáles son las formas más usuales de colaboración y cuál es el rol de las TIC en el trabajo colaborativo teniendo en cuenta el contexto particular de los casos descriptos. Las principales conclusiones están asociadas al uso de los espacios de socialización y reflexión sobre las prácticas en los centros estudiados y a la manera en que los docentes y la dirección pueden aprovechar esas instancias para el intercambio sobre aspectos ligados a lo pedagógico.

Palabras clave: colaboración docente, desarrollo profesional, gestión del conocimiento, TIC, estudio de casos

\begin{abstract}
Collaboration between teachers, with or without the use of information and communication technologies (ICT), is a determining factor in their professional development and, therefore, a key element for the proper functioning of educational centers and the quality of school system. For such reason, and given the paucity of research on the subject in Uruguay, the forms adopted by collaboration for professional purposes and the uses of ICT for said purposes were explored. The study design followed the multiple case-study strategy for which four primary schools and four secondary schools were selected in the department of Colonia. At each center, an in-depth interview was conducted with the principal and two teachers identified for their attitudes toward collaboration from a discussion group conducted by teachers. In addition, context data were collected and non-participant observations of infrastructure and school environment were carried out. The data were processed according to a layer analysis protocol, in which two cases were described in detail. Patterns between these cases and cross-cutting themes were then sought. Finally, the findings were organized in a meta-matrix in order to show the links between what is identified as teacher collaboration, which are the most common forms of collaboration and what is the role of ICT in collaborative work, taking into account the particular context of the cases described. The main conclusions are associated with the use of spaces for socialization and reflection on the practices in the centers studied, and the way in which teachers and management can take advantage of these instances for the exchange on pedagogical aspects.
\end{abstract}

Keywords: teacher collaboration, professional development, knowledge management, ICT, case studies

\title{
Introducción
}

Transformada en una capacidad de desarrollo obligado en distintos ámbitos del quehacer humano en el siglo XXI, la colaboración es un término que refiere al trabajo conjunto entre personas para lograr un fin común. Diversos autores han señalado la importancia de la colaboración entre docentes para el desarrollo profesional (Castañeda \& Adell, 2011; Vaillant, 2010; Westheimer, 1999), para el impacto en la eficacia de los aprendizajes de los estudiantes y para la mejora del desempeño de los centros educativos (Kelchtermans, 2006; Westheimer, 2008) así como para el desarrollo organizacional basado en la creación de conocimiento (Gairín, Muñoz, \& Rodríguez-Gómez, 2009; Gairín \& Rodríguez-Gómez, 2013). También se ha expuesto su relevancia en el ámbito educativo al establecer a la colaboración como una habilidad crítica para la creación de una cultura organizacional de trabajo en conjunto que favorece la interacción entre docentes. No obstante, la colaboración solo puede darse de manera voluntaria, entre docentes en situación de paridad y cuando existen objetivos comunes. Cuando la colaboración se presenta, los recursos son compartidos y la responsabilidad por la toma de decisiones y por los resultados de la práctica en colaboración son asumidos por el grupo (Friend \& Cook, 2013). 
Sin embargo, una revisión de la literatura deja al descubierto un primer problema respecto del concepto de colaboración docente: parece haber una confusión conceptual referida al término. En este sentido como sinónimos de colaboración se utilizan las siguientes expresiones: equipos de maestros, comunidades profesionales de aprendizaje, comunidades de aprendizaje docente, equipos de aprendizaje docente y comunidades de práctica, entre otros, configurando un problema para definir qué actividades de la labor docente la constituyen (Kelchtermans, 2006; Vangrieken, Dochy, Raes \& Kyndt, 2015).

Las investigaciones sobre colaboración en el ámbito del trabajo docente se remontan a los años 80 y 90 (Fullan \& Hargreaves, 1991; Little, 1990; Rosenholtz, 1985, 1991, por citar algunos de los más trascendidos). Pero desde principios de este siglo el concepto se ha transformado en un cliché asociado al cambio educativo (Lavié, 2009) y existen variados estudios que refieren a la temática o a conceptos de uso asociado estableciendo una variedad de características y categorías de lo que comprende la colaboración y sus modalidades de aplicación en el trabajo docente (Barfield, 2016; Datnow, 2011; Kyndt, Gijbels, Grosemans \& Donche, 2016; Ostovar-Nameghi \& Sheikhahmadi, 2016; Ronfeldt, 2016; Ronfeldt, Owens, McQueen \& Grissom, 2015; Vangrieken et al., 2015).

A efectos de determinar aspectos del encuadre teórico sobre los que desplegar el diseño de esta investigación, se entiende que la colaboración docente con fines profesionales es un término paraguas que engloba diferentes aspectos del trabajo colaborativo. En tanto se define como la interacción grupal en las actividades necesarias para completar una tarea compartida, también se asume como un constructo dinámico donde diferentes tipos de colaboración pueden ocurrir en diversos grados de profundidad (Vangrieken et al., 2015). En este contexto surge la necesidad de conocer cómo se desarrolla el trabajo colaborativo entre docentes en Uruguay, donde se hace evidente la falta de estudios relacionados con la temática y se cree oportuno realizar aportes al respecto que contribuyan a la mejora de los aprendizajes y al perfeccionamiento profesional del profesorado. A la vez, se considera acertado explorar las posibilidades que brindan los recursos TIC provistos por el Plan Ceibal desde 2008 de forma universal en la educación pública del país, en relación al desarrollo del trabajo colaborativo en el ámbito profesional de los educadores.

En especial, se indaga en las formas de trabajo colaborativo asociadas a la virtualidad y, por tanto, se sondean las actitudes de los docentes frente a las tecnologías de la información y la comunicación (TIC) para determinar los usos profesionales dentro y fuera del centro (Drossel, Eickelmann \& Gerick, 2017; Meneses, Fàbregues, Rodríguez-Gómez \& Ion, 2012; Suárez, Almerich, Díaz \& Fernández, 2012).

\section{Metodología}

Como estrategia de investigación se optó por un estudio de caso múltiple (Miles \& Huberman, 1994; Stake, 2006; Yin, 2014) adecuado para explorar y describir la compleja realidad educativa (Sabariego \& Bisquerra, 2009) y dar relevancia a la opinión de los informantes sobre el tema (Bell, 2005) según el enfoque cualitativo.

Para la selección de los casos se definió como población objetivo (Fraenkel \& Wallen, 2009) a los centros públicos de Uruguay en el nivel de enseñanza primaria y media básica, donde todos los docentes fueron provistos con dispositivos TIC por el Plan Ceibal con el objetivo de poder identificar instancias de colaboración presencial y en línea tanto dentro como fuera del centro educativo. Luego se definió el universo de casos posibles acotando el rango a aquellos centros accesibles a nivel territorial (Stake, 2006). Como consecuencia de esta delimitación se formuló un marco de muestreo de la totalidad de escuelas primarias y liceos con ciclo básico del departamento de Colonia (Crano, Brewer \& Lac, 2015). 
Los criterios de selección atienden a la oportunidad que brindan los casos para aprender sobre el fenómeno, independientemente de que sean casos típicos o no, tal como recomienda Stake (1995).

Se adoptó entonces, el criterio de máxima variación entre casos de las variables de interés, consideradas clave para entender los procesos de colaboración en contexto (Flyvbjerg, 2006; Miles \& Huberman, 1994; Patton, 2002; Yin, 2014). Los casos con las condiciones teóricas extremas y opuestas fueron elegidos para el estudio: nivel de contexto sociocultural y tasa de repetición del centro (ver Tabla 1).

Tabla 1. Características de los casos para el estudio.

\begin{tabular}{|c|c|c|c|c|c|}
\hline Caso & Nivel & $\begin{array}{l}\text { Nivel de contexto } \\
\text { socio - cultural }\end{array}$ & $\begin{array}{l}\text { Tasa de } \\
\text { repetición }\end{array}$ & Tipo de centro ${ }^{2}$ & Código \\
\hline 1 & \multirow{4}{*}{ Primaria } & Quintil 1 & Mínima & Escuela urbana, Tiempo extendido & $\mathrm{C} 1$ \\
\hline 2 & & Quintil 1 & Máxima & Escuela urbana, A. PR. EN. D.E.R & $\mathrm{C} 2$ \\
\hline 3 & & Quintil 5 & Mínima & Escuela urbana, De Práctica docente & $\mathrm{C} 3$ \\
\hline 4 & & Quintil 5 & Máxima & Escuela urbana, Común & C4 \\
\hline 5 & \multirow{4}{*}{ Secundaria } & Quintil 4 & Mínima & Liceo, urbano & $\mathrm{C} 5$ \\
\hline 6 & & Quintil 3 & Máxima & Liceo, rural & C6 \\
\hline 7 & & Quintil 5 & Mínima & Liceo, urbano & C7 \\
\hline 8 & & Quintil 5 & Máxima & Liceo, urbano & $\mathrm{C} 8$ \\
\hline
\end{tabular}

Notas:

${ }^{1} T a s a$ de repetición en relación al nivel de contexto socio - cultural

${ }^{2}$ Tipo de centro resultante luego de la selección

Fuente: Elaboración propia en base a datos proporcionados por CEIP y CES.

En cuanto a la selección de informantes se tuvo en cuenta el rol en el centro y la actitud frente a la colaboración. Se aplicó la técnica grupo de discusión que consistió en que en cada centro se pidiera la opinión a un grupo de docentes sobre aspectos de la colaboración desarrollada en ese lugar en particular. Los ítems de la pauta sondeaban el ideario de la colaboración y las características del docente que colabora; los aportes de las TIC para el proceso de colaboración, el uso de estos recursos para el intercambio con colegas; aspectos del centro que promueven la colaboración, y el registro y la socialización de experiencias dentro y fuera del centro.

Los informantes del grupo de discusión participaron voluntariamente (ver Tabla 2, para el detalle de la composición del grupo en cada centro) e identificaron a dos docentes que, en opinión del grupo, correspondiesen al perfil de docente colaborador y de docente autónomo (o individualista en el colectivo docente). 
Tabla 2. Participantes y tiempo de duración de la aplicación de la técnica de grupo de discusión.

\begin{tabular}{|c|c|c|}
\hline Caso & Nivel & Tiempo* \\
\hline 1 & 7 & 70 \\
\hline 2 & 4 & 75 \\
\hline 3 & 4 & 50 \\
\hline 4 & 4 & 65 \\
\hline 5 & 5 & 80 \\
\hline 6 & 5 & 75 \\
\hline 7 & 4 & 55 \\
\hline 8 & 5 & 70 \\
\hline Promedio & $\mathbf{5}$ & $\mathbf{6 8}$ \\
\hline
\end{tabular}

*Tiempo aproximado en minutos.

Fuente: Elaboración propia.

Luego se aplicaron entrevistas en profundidad a los docentes señalados y al director en cada centro, quien también informó sobre datos del contexto del caso (ver Tabla 3). Los ítems de las entrevistas siguieron los mismos ejes de la propuesta al grupo de discusión, profundizando en las experiencias y opiniones sobre la colaboración y los aspectos del trabajo en el centro que la favorecen o desestimulan.

Tabla 3. Duración de la aplicación de entrevistas en profundidad según el informante y el caso.

\begin{tabular}{|c|c|c|c|}
\hline \multirow{2}{*}{ Caso Informante } & \multicolumn{2}{|c|}{ Director } & Docente colaborador \\
\cline { 2 - 4 } & \multicolumn{3}{|c|}{ Tiempo* } \\
\hline 1 & 55 & 40 & 40 \\
\hline 2 & 60 & 120 & 55 \\
\hline 3 & 55 & 60 & 100 \\
\hline 4 & 60 & 50 & 60 \\
\hline 5 & 180 & 60 & 45 \\
\hline 6 & 60 & 75 & 40 \\
\hline 7 & 110 & 75 & 45 \\
\hline 8 & 120 & 70 & $\mathbf{5 7}$ \\
\hline
\end{tabular}

*Tiempo aproximado en minutos.

Fuente: Elaboración propia.

En cada visita al centro se recogieron observaciones e impresiones que sirvieron para contextualizar al centro y caracterizarlo en relación a aspectos de infraestructura, ubicación, ambiente, entre otros. Se codificaron según el código del centro y el número de observación (por ejemplo: C1_O1 para el caso de la primera observación en el caso 1). Para el análisis de datos se desarrolló un protocolo con el fin de dar transparencia y confiabilidad a la investigación (Yin, 2014). La codificación de la evidencia tuvo dos fases: una primera etapa donde se aplicaron códigos definidos con anterioridad según los objetivos de la investigación y se reconocieron códigos empíricos en las transcripciones del caso 1 (caso 
piloto), y una segunda etapa en la que se realizó una codificación enfocada que permitió el desarrollo de categorías analíticas o agrupaciones de códigos (Bazeley, 2013; Miles \& Huberman, 1994; Saldaña, 2009).

Así, a partir de las dimensiones de análisis se estableció el libro de códigos definitivo que se aplicó a la evidencia del caso 8 para comprobar su adecuación y aplicabilidad entre casos. A cada dimensión de análisis se la codificó tal como sugieren Miles y Huberman (1994) combinando códigos con las categorías y subcategorías de análisis que, a su vez, se relacionan con los propósitos de la investigación (ver Tabla 4).

Tabla 4. Extracto de Libro de códigos utilizado.

\begin{tabular}{|c|c|c|c|c|}
\hline \multirow[b]{2}{*}{1.} & \multirow{2}{*}{$\begin{array}{l}\text { Categorías y sub-categorías analíticas } \\
\text { Elementos caracterizadores del centro }\end{array}$} & \multicolumn{3}{|c|}{ Códigos } \\
\hline & & EC & - & - \\
\hline 1.1 & Ciudad / Zona en la que se localiza el centro & EC & CSC & - \\
\hline 1.2 & Nivel de contexto socio - cultural (Quintil) & EC & Mínima & \\
\hline$\ldots$ & $\ldots$ & $\ldots$ & $\ldots$ & $\ldots$ \\
\hline 1.8 & Desempeño del centro & $\mathrm{EC}$ & DES & - \\
\hline 1.8 .1 & Tasa de repetición baja & $\mathrm{EC}$ & DES & $\mathrm{B}$ \\
\hline 1.8 .2 & Tasa de repetición alta & EC & DES & A \\
\hline 2. & Organización del centro & OC & - & - \\
\hline 2.1 & Facilitadores para la colaboración en el centro & $\mathrm{OC}$ & FAC & - \\
\hline 2.1 .1 & Intrínsecos & $\mathrm{OC}$ & FAC & FAC_INT \\
\hline 2.1 .2 & Extrínsecos & $\mathrm{OC}$ & FAC & FAC_EXT \\
\hline$\ldots$ & $\ldots$ & $\ldots$ & $\ldots$ & $\ldots$ \\
\hline 2.6 & Clima organizacional para la colaboración & OC & CLI & - \\
\hline 2.6 .1 & Favorable & $\mathrm{OC}$ & $\mathrm{CLI}$ & FAV \\
\hline 2.6 .2 & Neutro & $\mathrm{OC}$ & $\mathrm{CLI}$ & NEU \\
\hline 2.6 .3 & Desfavorable & $\mathrm{OC}$ & $\mathrm{CLI}$ & DES \\
\hline 2.7 & Supervisión & $\mathrm{OC}$ & SUP & - \\
\hline 2.7 .1 & Visión desde el punto de vista de los docentes & $\mathrm{OC}$ & SUP & PVD \\
\hline 3. & Colabración docente & $C D$ & - & - \\
\hline 3.1 & Definición de colaboración & $C D$ & DEF & - \\
\hline 3.1 .1 & Criterios amplios & $C D$ & DEF & AMP \\
\hline 3.1 .2 & Criterios específicos & $C D$ & DEF & ESP \\
\hline$\ldots$ & $\ldots$ & $\ldots$ & $\ldots$ & $\ldots$ \\
\hline 3.5 & Dificultades asociadas & $C D$ & DIF & - \\
\hline 3.5 .1 & Organizacionales & $C D$ & DIF & ORG \\
\hline 3.5 .2 & Personales & $C D$ & DIF & PER \\
\hline 4. & Uso de TIC & UT & - & - \\
\hline 4.1 & Actitud frente a las TIC & UT & $\mathrm{ACT}$ & - \\
\hline 4.1 .1 & Abierta & UT & $\mathrm{ACT}$ & $\mathrm{ABI}$ \\
\hline 4.1 .2 & Neutra & UT & $\mathrm{ACT}$ & NEU \\
\hline$\ldots$ & $\ldots$ & $\ldots$ & $\ldots$ & $\ldots$ \\
\hline 4.4 .2 & Desventajas o problemas percibidos & UT & PCE & - \\
\hline 4.4.2.1 & Relacionadas a los dispositivos y la conectividad & UT & PCE & - \\
\hline 4.4.2.2 & Relacionadas a otros aspectos del programa & UT & PCE & - \\
\hline
\end{tabular}

Fuente: Elaboración propia. 
El análisis se realizó en cuatro etapas o capas (ver llustración 1). Primero, se hizo foco en la unidad de análisis. Los datos fueron examinados en profundidad y se organizaron en una descripción completa que da cuenta de la integralidad del caso. Una vez llevado a cabo el desarrollo completo de los casos 1 y 8 se pusieron en práctica comparaciones preliminares entre casos en busca de patrones, tal como indica la segunda capa de análisis (Chmiliar, 2010; Miles \& Huberman, 1994; Stake, 1999).

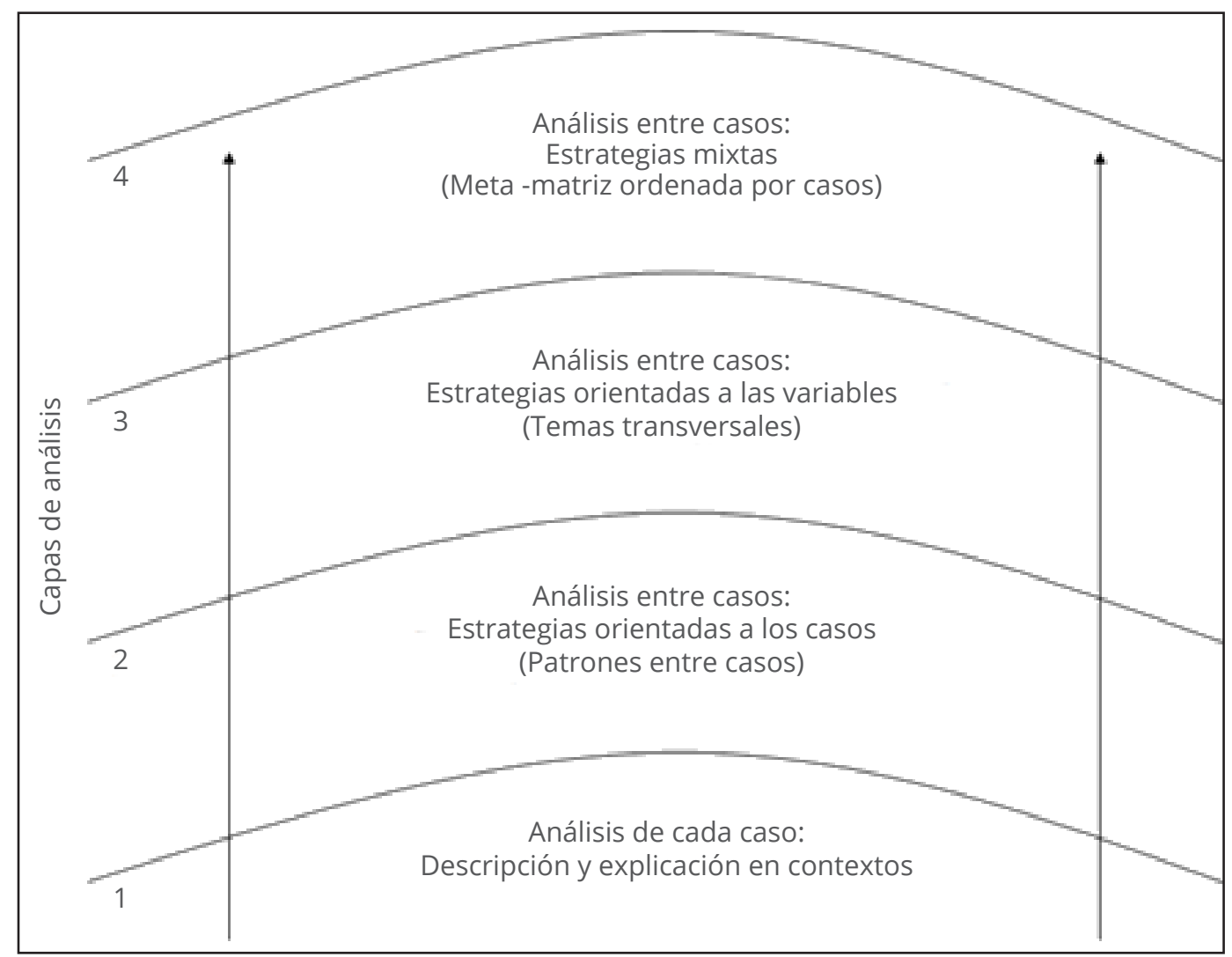

Ilustración 1. Desarrollo del proceso del análisis de datos.

Fuente: Elaboración propia en base a Miles \& Huberman (1994).

En una tercera fase o capa de análisis los datos se examinaron a través de los casos para identificar si existía algún patrón de variables o temas que trascendiesen a cada unidad. En dicho análisis se identificaron patrones, características comunes, elementos esenciales y componentes intervinientes (Chmiliar, 2010; Miles \& Huberman, 1994; Yin, 2014). Las similitudes subyacentes y constantes mostraron explicaciones más generales. Por último, la cuarta capa consistió en integrar los resultados de capas previas, es decir, la estrategia de examen de casos se combinó con la de examen del comportamiento de las variables entre casos en una meta-matriz como el resultado de la aplicación de técnicas mixtas de análisis.

Con lo antes expuesto y como recomienda Yin (2014), el diseño del análisis es fundamental para establecer un protocolo que guíe la lógica del examen y la presentación de los hallazgos. Mediante la documentación de las estrategias el diseño gana más fuerza por las posibilidades de réplica y es posible lograr la generalización, a la vez que permite discernir los hallazgos planeados de aquellos debidos a la casualidad. 


\section{Resultados}

En esta sección se desarrollan los hallazgos a partir de la aplicación del protocolo de análisis antes mencionado. Cabe destacar que se trata de resultados preliminares de la investigación donde se analizaron y compararon, según las capas propuestas en el protocolo, las características de los casos 1 y 8 en su contexto, los patrones entre casos, los temas transversales a las unidades de análisis y, por último, se organizaron los hallazgos y se estudiaron a partir del análisis de la meta-matiz.

\section{Caso 1: Escuela de quintil 1, con alto desempeño}

El centro es una escuela urbana que funcionaba en modalidad de tiempo extendido (de 10 a 17 horas) atendiendo a 159 estudiantes de un barrio de la periferia de la ciudad y de un asentamiento irregular, en un edificio bien mantenido pero poco adecuado a las necesidades de espacio. La directora, asistida por una secretaria, lideraba un grupo de ocho maestras y cuatro docentes encargados de talleres (arte, deportes, informática e inglés), en su mayoría radicados en la ciudad y estables en el centro desde 2013.

El involucramiento de las familias era bajo y los problemas de los alumnos notorios. Falta de documentación y seguimiento de controles de salud, problemas de aprendizaje y conductas disruptivas o violentas eran los emergentes con los que trataban las maestras en la escuela a diario, lo que implicaba un rendimiento medio-bajo de los estudiantes a nivel académico. A pesar de que algunos estudiantes de clases superiores tuviesen problemas para escribir o leer, las estadísticas de desempeño se explicaban por la necesidad de egreso de alumnos con extra edad o presiones de la inspección para asegurar los pases de grado y mantener estándares determinados.

Los testimonios ilustran sobre la situación de los estudiantes que concurren a la escuela y son representativos de las derivaciones del trabajo en el centro:

"Siempre tenemos un tema urgente que tratar (...) son temas urgentes, de niños que (...) son violados, o cosas así..." (C1_EDC:98).

"Los [estudiantes] míos quieren ser (...) de grandes, aspiran a ser carnicero o recolector de basura, eso es lo que ellos quieren ser, por ejemplo... No aspiran a más nada..." (C1_FG:355).

"Consume mucho [tiempo] el problema de conducta" (C1_FG:55).

"Tienen problemas entre las familias y se vienen acá a la clase a discutir. Entonces el contexto afecta mucho, mucho los aprendizajes" (C1_EDC:23).

Como características de la organización promotoras de colaboración se apuntaban las decisiones acordadas y la existencia de un grupo proactivo de cuatro docentes (C1_EDC:60). Sin embargo aparecían como impedimentos para el trabajo en colaboración la agenda de las coordinaciones semanales impuesta por la supervisión y los requisitos y formalidades de la planificación exigidos por la inspección. Por estos motivos, la supervisión (dirección e inspección) era considerada por las docentes como carente de apoyo. Algunos entrevistados se manifiestan al respecto como sigue:

"Siempre estás pensando qué hacer, porque la directora es muy exigente" (C1_EDA:90). "Esto así está mal redactado... porque la inspectora lo quiere de esta forma" (C1_FG:105). "Cuando tenemos la coordinación es el único momento que nos encontramos todas, que decimos bueno, podemos hablar y comunicarnos (...) y muchas veces no tenemos ese espacio, la mayoría de las veces, ¿por qué? Porque viene la inspectora y le dice: 'No, porque en la coordinación se tiene que trabajar algo de esto, de esto y de esto'" (C1_FG:318). 
A su vez, la existencia de un grupo que llevaba adelante los trabajos en colaboración y docentes que no participaban en estos proyectos sumado a un liderazgo percibido como negativo que generaba divisiones y tensión entre las docentes y resentía el vínculo con la dirección disminuía las posibilidades de colaboración del colectivo. En este sentido, los testimonios muestran el descontento con las exigencias y el desestímulo al trabajo en colaboración:

“Yo haría muchas más actividades con compañeras si no me pidieran la planificación, pero si yo para coordinar con una compañera una actividad que te la explico, la hacemos, me piden tres hojas de planificación, me quedo en mi salón tranquila, lo hago y no me muevo a otro salón" (C1_FG:373).

"Casi todos los recursos y todo, salen de nosotros. Nosotros compramos las cosas, nosotros hacemos esto, nosotros, nosotros y nosotros. Más que nada las maestras que siempre andamos" (C1_EDC:60).

En cuanto a la socialización y reflexión dentro del centro, las docentes declaraban que se daba en los recreos, es decir, en espacios informales y no frecuentes. Fuera del centro el panorama no era mejor: las docentes no reconocían espacios formales de socialización y reflexión aunque la docente colaboradora mencionaba la participación en salas docentes virtuales y algunas de las maestras intercambiaban ocasionalmente con colegas de generación o familiares docentes. Los registros de las prácticas y, en especial, de aquellas prácticas que implicaban colaboración entre colegas (como la planificación o los proyectos de ciclos) tenían una socialización baja, circunscripta a familiares que trabajaban en otros centros y colegas con quienes se compartía el ciclo en la propia escuela. Por ejemplo mencionan:

"Ahora estamos las clases chicas, tenemos que hacer un proyecto... paradas en los recreos" (C1_FG:328).

"Por ejemplo mi hermana es maestra de otra escuela y coordinamos juntas" (C1_FG:15). "Y coordinamos mucho con la maestra de acá, de quinto, que estamos con un proyecto de ciclo" (C1_EDC:23).

Consultadas acerca de lo que implica la colaboración, las informantes coincidían en mencionar que consistía en el intercambio de ideas y experiencias pedagógicas que tenía como consecuencia directa el fortalecimiento profesional y la actualización en contenidos y técnicas de enseñanza, si bien no mencionaron beneficios a nivel del alumnado. Para este grupo, las características de un docente que colabora eran, en lo personal, la apertura al diálogo y el compañerismo, y en cuanto a lo profesional el apoyo y complementación (ver Tabla 5). 
Tabla 5. Elementos que integran el perfil del docente colaborador, Caso 1.

\begin{tabular}{|l|l|}
\hline Elementos personales & Elementos profesionales \\
\hline Compañerismo (C1_EDA: 146) & Compromiso con lo profesional (C1:EDi: 177) \\
\hline Piensa en el bien general y no en sí mismo (C1_EDC: 25) & $\begin{array}{l}\text { Preocupación por el aprendizaje de los alumnos } \\
\text { (C1_EDi: 177) }\end{array}$ \\
\hline Abierto (C1_FG: 51; C1_EDC: 9) & Complementación entre colegas (C1_EDA: 146) \\
\hline No teme plantear dudas, pedir ayuda (C1_EDC: 21, 51) & $\begin{array}{l}\text { Unidad en el colectivo para el logro del } \\
\text { aprendizaje de los alumnos (C1_EDC: 114) }\end{array}$ \\
\hline Ayuda (C1_FG: 36, 41,68; C1_ED: 9) & Comparte experiencias pedagógicas (C1_FG:9,36) \\
\hline Se comunica con los demás (C1_EDC: 21) & Apoya al colega (C1_FG: 9,10,68) \\
\hline
\end{tabular}

Fuente: Elaboración propia.

En este colectivo las experiencias de colaboración a nivel institucional (institucióninstitución, institución-comunidad) se reducían a visitas a liceos y escuelas técnicas con estudiantes de sexto año y cierres de talleres o actos culturales. A nivel pedagógico (trabajo coordinado por dos o más docentes del centro), se listaban la desestructuración de clases (clases compartidas) y los proyectos de ciclo. Asimismo, identificaban como colaboración al cambio de clase momentáneo de alumnos con problemas de conducta:

"Yo igual colaborar lo veo más como ayudar cuando pasa algo (...) puntual..." (C1_FG:36); "frente a una dificultad, algo que sale de lo común, desajustes de conducta" (C1_FG:38, $39,43,48)$.

Las experiencias a nivel institucional fuera del centro estaban a cargo de la maestra colaboradora, quien organizaba la participación en la Feria Ceibal y había publicado sus experiencias en una revista. A nivel pedagógico la interacción que implicaba colaboración se daba con familiares docentes, conocidas o colegas de otros centros en los que trabajaban al momento del estudio, de manera principal para planificar, coordinar actividades de grupos por videoconferencia y en menor medida para intercambiar sobre algún tema puntual. De las ocho docentes consultadas solo dos declararon realizar este tipo de actividades. Las causas de la baja colaboración fueron asociadas a aspectos personales como el egoísmo de las colegas. Así refiere la docente colaboradora:

“Para mí el egoísmo de ciertas compañeras es... Ahhh! no se puede trabajar. Ahora estamos por ejemplo por hacer un... el cierre de talleres, pero hay dos compañeras que no quieren saber nada y como que siempre están trancando y poniendo piedras en el camino. Para mí eso es egoísmo, ¿viste? Porque vos tenés que pensar un poco en los chiquilines también, no en tus horarios" (C1_EDC:25).

En este grupo se utilizaban los documentos en la nube para colaborar sólo en los proyectos de ciclo y la mensajería instantánea para cuestiones administrativas o de organización. Según la directora, sólo cuatro de las ocho docentes utilizaban recursos TIC para el trabajo y afirmaba que dos maestras aún planificaban en manuscrita y una de ellas se resistía a la incorporación de TIC. Sin embargo, solo dos docentes parecían conocer, reconocer como herramienta idónea para la colaboración y utilizar las plataformas educativas del Plan Ceibal (ver Tabla 6). 
Tabla 6. Herramientas TIC según el conocimiento y uso dados por los docentes, Caso 1.

\begin{tabular}{|c|c|c|}
\hline & Herramientas para el aula & Herramientas para colaborar \\
\hline $\begin{array}{l}\text { Conocidas } \\
\text { (no usadas) }\end{array}$ & & - Correo (C1_EDi: 104, 122) \\
\hline Usadas & $\begin{array}{l}\text {-Plataformas educativas (C1_FG: 254) } \\
\text {-Plataformas específicas de asignatura } \\
\text { (C1_EDC: } 31,35,43 \text { ) } \\
\text {-Aplicaciones para presentaciones (C1_FG: 273) } \\
\text { - Aplicaciones de videoconferencia (C1_EDi: 11; } \\
\text { C1_FG:27) }\end{array}$ & $\begin{array}{l}\text {-Almacenamiento compartido en la } \\
\text { nube (C1_EDC: 49) } \\
\text {-Mensajería instantánea (C1_FG: } 202 \\
\text { 204; C1_EDA:68) } \\
\text {-Plataformas educativas (C1_FG:194, } \\
\text { 195, 287, 291, 293) }\end{array}$ \\
\hline
\end{tabular}

Fuente: Elaboración propia.

Las actitudes frente a las TIC se catalogaban de neutras para la mayoría de las informantes posiblemente a causa de las desventajas asociadas a los dispositivos Ceibal: falta de reparación, reposición y asignación de dispositivos para las docentes. Esto, sumado a la falta de cuidados de los dispositivos por parte de los estudiantes y sus familias propiciaba problemas operativos en el aula para el uso de los equipos, las plataformas y los recursos educativos. En relación a los dispositivos y las dificultades asociadas comentan:

“Es porque o ellos la dejan en la casa, o la traen sin cargar, o está rota, o no sé... entonces claro, vos vas a la clase donde tenés veinte alumnos, cuando tenés seis computadoras" (C1_FG:209).

"Ayer ganas de llorar de ver una tablet que le habían sacado la pantalla. Entonces vos decís: '¿Cómo es posible esto que no lo cuiden?'" (C1_FG:221).

"No hay un uso responsable..." (C1_FG:208).

En síntesis, el trabajo en el centro se veía influido de forma negativa por el contexto donde se instala el centro y las características de las familias que atendía, dificultando los aprendizajes de los estudiantes. La modalidad de tiempo extendido intentaba mejorar la situación de algunos estudiantes menos favorecidos, pero la violencia y el conflicto del contexto, trasladado al aula, dificultaba aún más los logros de aprendizaje esperados debido a la escasez de tiempo pedagógico.

El espacio de coordinación inadecuado a criterio de las docentes y la visión de la supervisión como un elemento distorsionante del clima organizacional y de las iniciativas de trabajo en equipo condicionaban la colaboración. Los intentos de colaborar se reducían a la coordinación impuesta de proyectos de ciclo, a actividades puntuales de la escuela o al intercambio de estudiantes con conductas disruptivas de modo de evitar el papeleo de la planificación adicional que implicarían otras actividades en colaboración. Dada la actitud mayormente neutra hacia las TIC, los recursos digitales para la colaboración eran poco explotados, en especial las herramientas provistas en las plataformas docentes. El contexto desfavorable a la colaboración y al desempeño de los estudiantes no se veía reflejado en las tasas de repetición del centro, según una docente, por el impacto de las políticas del subsistema para mejorar las estadísticas de rendimiento de primaria.

\section{Caso 8: Liceo de quintil 5, con bajo desempeño}

Este caso se trata de un centro urbano que funcionaba en el turno matutino al que concurrían 98 estudiantes provenientes de la localidad y de la zona rural adyacente, en un edificio nuevo, con una infraestructura adecuada para el desarrollo de las actividades educativas. La directora, en el cargo por primera vez en 2016, tenía bajo su supervisión a un grupo de 25 docentes, una adscripta y una secretaria. Una parte de los docentes era 
estable, provenientes de la localidad o de una localidad cercana, mientras que el resto viajaba desde otros puntos del departamento y rotaba de año a año lo que dificultaba su sentido de pertenencia al liceo.

Por un lado, las familias de los estudiantes tenían mayormente alto nivel de involucramiento con el seguimiento de los trayectos académicos y participaban activamente de los eventos propuestos por el centro. Un porcentaje no se involucraba en absoluto y coincidía con los estudiantes de bajo rendimiento, que eran pocos, y a los que se realizaba un seguimiento con clases extra de materias específicas. Sin embargo, a criterio de la directora el impacto de la repetición o el abandono de pocos estudiantes impactaba las estadísticas del desempeño general del centro elevando la tasa de repetición de manera notoria. Por otro lado, un docente mencionaba que, dadas las características del liceo los estudiantes no podían seguir ciertos cursos, lo que desestimulaba la continuidad en el sistema educativo debido a la necesidad de trasladarse a otras localidades para proseguir los estudios.

A pesar de estas cuestiones la directora y un docente marcaban que los estudiantes, en general, tenían un nivel de desempeño medio-alto y se mostraban comprometidos con sus aprendizajes:

"Tienden a la perfección como estudiantes" (C8_EDi:13) y “Los trabajos que salen, la verdad que te asombran (...) donde se ponen las pilas los chiquilines, la creatividad que tienen es fabulosa (...) continuamente tengo que estar buscando cosas nuevas para que ellos puedan trabajar" (C8_EDC:34).

Entre los elementos de la organización del centro que propiciaban la colaboración se destacaban las estrategias aplicadas por la dirección, en especial la apertura hacia las propuestas y la flexibilidad en el trabajo. En concordancia con estos facilitadores el clima era visto como agradable y cómodo y los docentes se sentían apoyados por la dirección. En la perspectiva del docente colaborador se rescata este testimonio:

“El apoyo que brinda la dirección, para sea el trabajo que sea que se plantea, es fundamental. Acá se apoyan los proyectos que puedan surgir, los trabajos colaborativos que puedan surgir, siempre y cuando, lógicamente, nos atengamos a lo que es la reglamentación (...) Pero el apoyo de la dirección es fundamental. Y acá se da" (C8_ EDC:95).

Por otra parte, como contrapesos a la colaboración se enumeraban la escasez de medios de transporte en la localidad y los horarios limitados de frecuencias que, sumados al multiempleo de algunos docentes, hacía imposible la concreción de instancias de coordinación, más allá de las establecidas por el sistema con periodicidad semanal. Los testimonios de los informantes reflejan algunas de las complicaciones del multiempleo:

"Cuando tenés un liceo, dos liceos, tres liceos, cuatro liceos. Con cuál me comprometo, con cuál... Si te comprometés con todos te volvés loco" (C8_FG:83).

"La mayoría corren de un liceo para otro ¿y para qué? Para comer, en definitiva. Digo, para mantener una familia, una casa" (C8_EDi:76).

Las opiniones respecto de la socialización y reflexión de prácticas colaborativas dentro del centro variaban dependiendo de la carga horaria del informante en el liceo. Aquellos docentes con menor carga horaria no visualizaban la coordinación como un espacio de intercambio docente, mientras que profesores con mayor carga horaria entendían que se trataba de un espacio de socialización que se prestaba a la reflexión sobre las prácticas y 
al trabajo en colaboración. Esto se explicaba teniendo en cuenta que la cantidad de horas asignadas a coordinación dependía de la carga horaria total en el centro.

Fuera del liceo, la socialización se asumía como baja; solo un docente menciona los cursos en línea como medio de socialización de las prácticas de aula y no se reconocían espacios formales dedicados a tal fin. En cuanto a la reflexión se identificaban las salas docentes departamentales o nacionales de carácter anual o intercambios con colegas de periodicidad variable para intercambio de contenidos o planificación. Los registros formales de las prácticas se realizaban en el libro del profesor y no se compartían con otros docentes; registros discrecionales, como proyectos en conjunto no eran realizados, o bien eran realizados pero no socializados con otros colegas. Dos testimonios refieren a estos temas:
"Salas docentes que se hacen una vez cada tanto..." (C8_EDA:216).
"Esa experiencia registrada queda en la libreta. En el desarrollo del curso del docente, en mi caso" (C8_EDC:121).
“Todos estos cursos que estamos haciendo son cursos en sí, de forma colaborativa. Si bien los podés hacer de forma individual pero te piden que algo hagas colaborativamente" (C8_EDC:57).

Los informantes de este liceo entendían la colaboración como un compromiso por parte del docente con la formación integral del estudiante y de allí se desprendían tareas o actividades enfocadas al aprendizaje de los alumnos como construcción conjunta de los docentes que beneficia a los estudiantes porque los motiva. En cuanto a las características profesionales del profesorado colaborador, los informantes identificaban la apertura al diálogo y la disposición a ayudar al colega (ver Tabla 7). Lo antes dicho servía para generar sinergias enriquecedoras entre los profesores, aunque se mencionaba como dificultad asociada a los procesos de colaboración el tiempo necesario para planificar.

Tabla 7. Elementos que integran el perfil del docente colaborador, Caso 8.

\begin{tabular}{|l|l|}
\hline Elementos personales & Elementos profesionales \\
\hline Compromiso (C8_FG: 80, 133, C8:EDi:13) & Enfoque en el estudiante (C8_FG: 89, 93) \\
\hline Capacidad de escucha y propuestas (C8_FG: 67) & $\begin{array}{l}\text { No limitado por el programa de su asignatura } \\
\text { (C8_FG: 93-96) }\end{array}$ \\
\hline Apertura al diálogo (C8_FG: 65) & Ayuda al colega (C8_EDA: 268) \\
\hline Brinda lo mejor de si mismo (DC: 155) & Comparte lo que hace bien (C8_EDi: 260) \\
\hline da, aporta (C8_EDA: 268, EDC: 29, 155) & $\begin{array}{l}\text { Claridad sobre la finalidad de la función docente } \\
\text { (C8_EDi:260) }\end{array}$ \\
\hline Sentido común (C8_FG: 64) & Se comunica con otros docentes (C8_FG: 23) \\
\hline
\end{tabular}

Fuente: Elaboración propia.

Por una parte, en este centro las experiencias de colaboración a nivel institucional (institución-institución; institución-comunidad) eran numerosas, realizándose eventos benéficos, actos, talleres y representaciones. En lo relativo a lo pedagógico, se generaban evaluaciones y actividades compartidas entre docentes de materias diferentes que se proponían a los estudiantes, pero no de manera usual. Por otra parte, entre docentes, se organizaban talleres para el aprendizaje de las prestaciones de recursos TIC como por ejemplo manejo de plataformas educativas y aplicaciones para presentaciones presentados a los docentes durante la coordinación por parte del profesor colaborador, tal como se relata a continuación: 
"Sí, en el sentido de darles este año, dos cursos en sí, para el manejo de la plataforma Edmodo y el manejo de Prezi. Que eso nació de mí, y se lo planteé a la directora para darlo en coordinación y lo dimos en... fueron dos coordinaciones. En este caso, que dediqué, o sea, dos coordinaciones a Edmodo y dos coordinaciones a Prezi. La idea mía era plantear otro taller, pero ya la altura en que estamos como que no... No me da más" (C8_EDC:9).

Fuera del centro las experiencias de colaboración a nivel institucional partían de pedidos de la dirección para organizar actividades con liceos de otras localidades. Las experiencias a nivel pedagógico se realizaban de forma preponderante y más o menos sistemática, motu proprio con colegas de generación o consistía en comunicaciones esporádicas. Las excusas que surgían de forma recurrente como barrera a la colaboración era el tiempo extra necesario para la planificación y las escasas posibilidades de locomoción para viajar a la localidad. Una síntesis sobre la colaboración en el centro se resume en el testimonio de uno de los docentes entrevistados:

“Uno mismo se mete en su trabajo, y no es que no quiera compartir sino que no está habituado a compartir. Porque veo también que los docentes, sea cual sea la asignatura, los centros, sea cual sea el centro, no estamos, me voy a incluir, acostumbrados a colaborar entre nosotros y a difundir lo que hacemos. ¿Sí? No estamos acostumbrados a trabajar colaborativamente todavía, al cien por ciento. (...) Pero que tampoco podés obligar (...) esto no es cuestión de obligar a nadie sino es cuestión de concientizarse. De que lo mejor es el trabajo colaborativo hoy en día y de que te puede enriquecer a vos. No solo al alumno le sirve, sino también a uno, como docente en sí" (C8_EDC:141).

Según el grupo de informantes las herramientas TIC utilizadas para la colaboración se remitían al uso de mensajería instantánea y correo electrónico, mientras que la mayoría de los docentes declaraba no utilizar a menudo plataformas educativas para la colaboración o para el trabajo en el aula. Solo uno de los docentes, el identificado como colaborador, utilizaba documentos compartidos en la nube con sus estudiantes y declaraba utilizar una plataforma educativa con ellos (ver Tabla 8).

Tabla 8. Herramientas TIC según el conocimiento y uso dados por los docentes, caso 8.

\begin{tabular}{|l|l|l|}
\hline & Herramientas para el aula & Herramientas para colaborar \\
\hline $\begin{array}{l}\text { Conocidas } \\
\text { (no usadas) }\end{array}$ & $\begin{array}{l}\text { - Plataformas específicas de asignatura } \\
\text { (C8_EDA: 250,252) } \\
\text {-Aplicaciones de videoconferencia (C8_FG: 232) }\end{array}$ & $\begin{array}{l}\text { - Almacenamiento compartido en la } \\
\text { nube (C8_FG: 273-275, 282) } \\
\text { - Plataformas educativas (C8_EDC: 63) }\end{array}$ \\
\hline \multirow{5}{*}{ Usadas } & $\begin{array}{l}\text {-Almacenamiento compartido en la nube } \\
\text { (C8_EDC: 75) }\end{array}$ & \\
& $\begin{array}{l}\text { C8_FG:239) } \\
\text { - Plataformas educativas (C8_EDC: 87, 91; }\end{array}$ & $\begin{array}{l}\text { - Mensajería instántanea (C8_EDi: 112, } \\
114,116 ; \text { C8_EDA: 55; C8_FG:38, 391) } \\
\text {-Correo (C8_EDi:88) }\end{array}$ \\
\hline
\end{tabular}

Fuente: Elaboración propia.

Las actitudes frente a las TIC podían categorizarse, en general, como neutras debido a la falta de iniciativa para incorporar las herramientas al ámbito profesional o al trabajo de aula. En este sentido, se identificaban problemas asociados a los dispositivos e infraestructura provistos por el Plan Ceibal tales como problemas con la conectividad a Internet en el centro y dispositivos obsoletos que no habían sido reemplazados. En relación al Plan solo se marcó como ventaja la posibilidad de realización de cursos de capacitación en línea por parte del docente identificado como colaborador. Los testimonios indican: 
"La conexión a Internet está fallando. Está fallando mucho" (C8_FG:248).

"Hay docentes que están molestos en el sentido de que tienen computadoras que ya no les funcionan. Porque fueron las primeras que se entregaron y ya no funcionan" (C8_EDC:59). "Plan Ceibal, últimamente, está ofreciendo muchos cursos. Pero veo que no hay mucho involucramiento del docente respecto a Plan Ceibal. Para trabajar de forma colaborativa, en sí" (C8_EDC:57).

En resumen, el trabajo en el centro era favorecido por la directora, que intentaba generar sinergias de participación a pesar de las dificultades de acceso al centro y de la rotación docente que entorpecía el sentido de pertenencia a la institución. En este ambiente de trabajo los docentes se preocupaban por la formación integral de estudiante, apoyados en gran medida por las familias. Los casos de alumnos de bajo desempeño eran seguidos con clases de apoyo aunque el impacto de las repeticiones de grado era notorio en el desempeño general del centro.

La coordinación semanal reunía, en teoría, los elementos para generar la socialización y reflexión sobre las prácticas entre los docentes, pero los problemas de locomoción y el tamaño del liceo impedían encuentros con el suficiente tiempo para estos fines. La colaboración se reducía a cuestiones operativas, al funcionamiento del centro y a la organización de eventos institucionales, y en menor medida vinculaba a docentes con fines pedagógicos relacionados al trabajo del aula. A razón de la actitud neutra hacia las TIC de la mayoría de los docentes los recursos informáticos que habilitasen la colaboración eran poco utilizados (plataformas y documentos en la nube, por mencionar dos casos). En este contexto se hacían más notorios los esfuerzos del docente colaborador por capacitar a sus colegas en el uso de herramientas TIC que favoreciesen la colaboración y ahorrasen tiempo a los docentes.

\section{Análisis de patrones entre casos}

En esta capa de análisis se buscan similitudes o patrones entre casos, para lo que se confeccionó una tabla resumen con las principales características halladas en cada caso para cada categoría de análisis (ver Tabla 9). 
Tabla 9. Cuadro - síntesis comparativo de casos

\begin{tabular}{|c|c|c|c|c|}
\hline & & Categorías & Caso 1 & Caso 8 \\
\hline & & Ciudad/zona & Barrio periférico & Pequeña (1800 hab. est.) \\
\hline & & Contexto socio-cultural & Bajo (Q1) & Alto (Q5) \\
\hline & & Tipo de centro & Escuela urbana, tiempo extendido & Liceo urbano \\
\hline & 늘 음 인 & Edificio, capacidad & $\begin{array}{l}\text { Tamaño inadecuado, bien } \\
\text { mantenido }\end{array}$ & Tamaño adecuado, bien mantenido \\
\hline & 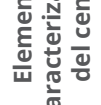 & Estudiantes & $\begin{array}{l}\text { Mayor proporción: nivel de } \\
\text { desempeño medio-bajo } \\
159 \text { estudiantes inscriptos }\end{array}$ & $\begin{array}{l}\text { Mayor proporción: nivel de } \\
\text { desempeño medio-alto } \\
98 \text { estudiantes inscriptos }\end{array}$ \\
\hline & & Familias & Acercamiento al centro: bajo & Acercamiento al centro: medio-alto \\
\hline & & Docentes & $\begin{array}{l}12 \text { docentes, en su mayoría de la } \\
\text { ciudad }\end{array}$ & $\begin{array}{l}25 \text { docentes, algunos de la } \\
\text { comunidad }\end{array}$ \\
\hline & & $\begin{array}{l}\text { Facilitadores para la } \\
\text { colaboración }\end{array}$ & $\begin{array}{l}\text { Decisiones acordadas /Grupo de } \\
\text { docentes activos }\end{array}$ & $\begin{array}{l}\text { Estrategias de dirección: apertura, } \\
\text { flexibilidad }\end{array}$ \\
\hline & & $\begin{array}{l}\text { Impedimentos para la } \\
\text { colaboración }\end{array}$ & $\begin{array}{l}\text { Utilización de coordinación / } \\
\text { Formalidades de planificación }\end{array}$ & $\begin{array}{l}\text { Accesibilidad del transporte / } \\
\text { Multiempleo }\end{array}$ \\
\hline & & $\begin{array}{l}\text { Socialización y reflexión en el } \\
\text { centro }\end{array}$ & $\begin{array}{l}\text { En coordinación: Baja /Espacios } \\
\text { informales: Media-baja }\end{array}$ & $\begin{array}{l}\text { En coordinación: Baja /Espacios } \\
\text { informales: Media-baja }\end{array}$ \\
\hline & 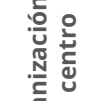 & $\begin{array}{l}\text { Socialización y reflexión fuera } \\
\text { del centro }\end{array}$ & $\begin{array}{l}\text { Espacios formales: No } \\
\text { Alternativas: Salas docentes, } \\
\text { colegas de generación }\end{array}$ & $\begin{array}{l}\text { Espacios formales: No } \\
\text { Alternativas: Salas docentes, } \\
\text { colegas de generación }\end{array}$ \\
\hline$\frac{\text { g }}{\frac{0}{n}}$ & 0 & $\begin{array}{l}\text { Registros de las prácticas } \\
\text { colaborativas }\end{array}$ & $\begin{array}{l}\text { Registros formales (planificación): } \\
\text { socialización baja } \\
\text { Registros formales (proyectos de } \\
\text { ciclo): socialización baja }\end{array}$ & $\begin{array}{l}\text { Registros formales (libreta): } \\
\text { socialización baja-nula } \\
\text { Registros discrecionales: } \\
\text { socialización baja-nula }\end{array}$ \\
\hline$\frac{\varepsilon}{a}$ & & Clima organizacional & $\begin{array}{l}\text { Cierta tensión, divisiones entre el } \\
\text { colectivo }\end{array}$ & Agradable, cómodo \\
\hline & & Supervisión & No apoya a docentes & Apoyo a docentes \\
\hline & & Definición & $\begin{array}{l}\text { Intercambio de ideas, experiencias } \\
\text { Fortalecimiento, actualización }\end{array}$ & $\begin{array}{l}\text { Compromiso con la formación } \\
\text { integral }\end{array}$ \\
\hline & $\begin{array}{l}\stackrel{ \pm}{c} \\
\stackrel{c}{ \pm} \\
\stackrel{\circ}{0}\end{array}$ & $\begin{array}{l}\text { Características del docente } \\
\text { que colabora }\end{array}$ & $\begin{array}{l}\text { Personales: Apertura, } \\
\text { compañerismo } \\
\text { Profesionales: Apoyo, } \\
\text { complementación }\end{array}$ & $\begin{array}{l}\text { Personales: Apertura } \\
\text { Profesionales: Enfoque en el } \\
\text { estudiante }\end{array}$ \\
\hline & $\frac{\sqrt{2}}{\frac{1}{2}}$ & Experiencias dentro del centro & $\begin{array}{l}\text { Institucionales: Baja /Pedagógicas: } \\
\text { Baja }\end{array}$ & $\begin{array}{l}\text { Institucionales: Alta /Pedagógicas: } \\
\text { Baja }\end{array}$ \\
\hline & $\frac{8}{\frac{0}{0}}$ & Experiencias fuera del centro & $\begin{array}{l}\text { Institucionales: Baja/ Pedagógicas: } \\
\text { Media }\end{array}$ & $\begin{array}{l}\text { Institucionales: Baja /Pedagógicas: } \\
\text { Baja }\end{array}$ \\
\hline & & Beneficios percibidos & $\begin{array}{l}\text { Profesionales: Medio / } \\
\text { Estudiantes: No mencionan }\end{array}$ & $\begin{array}{l}\text { Profesionales: Medio /Estudiantes: } \\
\text { Medio }\end{array}$ \\
\hline & & Dificultades asociadas & Egoísmo de colegas & Tiempo para la planificación \\
\hline & & Actitud & Mayoría neutra & Mayoría neutra \\
\hline & $\underline{\underline{E}}$ & Para colaborar & $\begin{array}{l}\text { Mensajería instantánea: bajo } \\
\text { Documentos compartidos en la } \\
\text { nube: medio }\end{array}$ & $\begin{array}{l}\text { Mensajería instantánea: bajo } \\
\text { Correo electrónico: bajo }\end{array}$ \\
\hline & $\frac{0}{0}$ & Para el aula & Plataforma educativa: medio-bajo & Plataforma educativa: medio-bajo \\
\hline & $\stackrel{\leftrightarrow}{\supset}$ & Plan Ceibal & $\begin{array}{l}\text { Ventajas: recursos digitales, } \\
\text { plataformas } \\
\text { Desventajas: falta de reparación, } \\
\text { recambio o asignación de } \\
\text { dispositivos }\end{array}$ & $\begin{array}{l}\text { Ventajas: capacitación online } \\
\text { Desventajas: falta de recambio, } \\
\text { problemas de conectividad en el } \\
\text { centro }\end{array}$ \\
\hline
\end{tabular}

Fuente: Elaboración propia. 
En cuanto a los elementos caracterizadores del centro se distingue que ambos son centros urbanos que funcionaban en edificios en buenas condiciones de mantenimiento y atendían a pocos estudiantes. Referido a la organización del centro existía un espacio de coordinación semanal que reunía a los docentes aunque en los dos centros las posibilidades de socialización y reflexión sobre las prácticas eran bajas en esos espacios, mientras que en espacios informales se percibía como media-baja de forma principal ligada a conversaciones en recreos o comentarios de pasillo. Otra similitud en cuanto a las cuestiones organizativas tiene que ver con la socialización y reflexión fuera del centro, para la que no se reconocen espacios formales y los informales se limitan a salas docentes organizadas por la inspección algunas veces al año pero no para todos los docentes.Las prácticas colaborativas realizadas en el centro se registraban en la planificación de cada docente, que solo era compartida con la dirección y, eventualmente, con la inspección por lo que la socialización era baja y discrecional con colegas. Los registros recogían las contadas experiencias de colaboración a nivel pedagógico, de manera principal dentro del centro y, en algunos casos, el bajo nivel de actividades en colaboración a nivel institucional.

Si se observan los beneficios del trabajo colaborativo que percibía el profesorado para su desarrollo profesional pueden interpretarse como de impacto medio, debido a que en ambos grupos declaraban que los enriquecía. Sin embargo, revisando las instancias de intercambio relatadas la puesta en práctica de la colaboración era limitada.

En ambos centros la actitud era neutra en cuanto al uso de TIC, con algunos pocos docentes que se destacaban por el manejo de herramientas y recursos y otros que estaban entre la negación y la apatía. Esto condicionaba la colaboración con colegas del centro fuera del espacio establecido que, como se mencionó, no podía utilizarse de manera concreta para el trabajo compartido.

En suma, atendiendo a las variables de interés para el estudio, colaboración y uso de TIC, por una parte se destacaba el poco uso de las herramientas digitales por parte de los docentes y su escasa aplicación al trabajo compartido. Por otra parte, se detectaban dificultades en cuanto a los dispositivos provistos por el Plan Ceibal a lo que se sumaban elementos de carácter personal tales como barreras a la colaboración.

\section{Análisis de temas transversales a los casos}

En este apartado se enfatiza en los hallazgos que trascienden a cada caso, destacando los elementos que tienen en común y señalando los factores que pueden interpretarse como causa de similitudes generales.

En primer lugar la coordinación docente, entendida como el espacio para el intercambio y reflexión sobre las prácticas, tenía escaso desarrollo desde el punto de vista pedagógico, siendo utilizada para temas del orden de lo administrativo y lo comunitario. La socialización de las prácticas se limitaba a espacios informales, también limitados por el poco tiempo disponible, ya fuera a causa de la locomoción o de los emergentes escolares.

En segundo lugar, las similitudes entre los centros a nivel de uso de TIC podían asociarse a la calidad de los dispositivos provistos por el Plan Ceibal. Sin embargo, los docentes que mostraban una actitud positiva hacia las TIC coincidían con los docentes más abiertos a la colaboración, por lo que podría pensarse que si bien el manejo de herramientas TIC es una ventaja para aprovechar el tiempo de trabajo en equipo y mejorar los resultados, la actitud hacia la colaboración es fundamental. 
En tercer lugar, aunque en el discurso general se entendía que la colaboración enriquecía a los docentes desde lo profesional, en ninguno de los casos se identificaron actividades claras para desarrollar instancias de trabajo compartido más allá de lo instrumental. El tiempo necesario para planificar y documentar las actividades y las actitudes de algunos docentes desestimulaba esta modalidad de trabajo.

Por último, no se identificaron líneas claras de acción desde la dirección para estimular la colaboración, si bien las directoras declaraban esfuerzos en la sensibilización de los docentes.

\section{Meta-matriz entre casos}

Como resultado del análisis de las capas anteriores, se presenta una matriz que sintetiza lo expuesto anteriormente (ver Tabla 10). Haciendo hincapié en el análisis de las variables de interés para el estudio también se señalan los patrones o elementos que presentan similitudes o diferencias entre los centros para arribar a conclusiones preliminares.

Tabla 10. Meta - matriz de análisis

\begin{tabular}{|c|c|c|c|}
\hline & Categoría & Caso 1 & Caso 8 \\
\hline \multirow{11}{*}{ 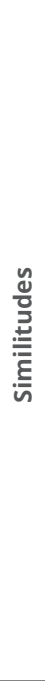 } & Tipo de centro & Escuela urbana & Liceo urbano \\
\hline & $\begin{array}{l}\text { Impedimentos para la } \\
\text { colaboración }\end{array}$ & Coordinación (agenda) & Coordinación (tiempo) \\
\hline & $\begin{array}{l}\text { Socialización/reflexión en el } \\
\text { centro }\end{array}$ & Baja & Baja \\
\hline & Socialización/reflexión fuera & Esporádica & Esporádica \\
\hline & $\begin{array}{l}\text { Registros de prácticas } \\
\text { colaborativas }\end{array}$ & Baja socialización & Baja socialización \\
\hline & $\begin{array}{l}\text { Experiencias dentro } \\
\text { (pedagógicas) }\end{array}$ & Baja cantidad & Baja cantidad \\
\hline & $\begin{array}{l}\text { Experiencias fuera } \\
\text { (institucionales) }\end{array}$ & Baja cantidad & Baja cantidad \\
\hline & Beneficios percibidos & Alto impacto & Alto impacto \\
\hline & Actitud frente a las TIC & Neutra & Neutra \\
\hline & TIC usadas para colaborar & Bajo impacto & Bajo impacto \\
\hline & Desventajas del Plan Ceibal & Dispositivos & Dispositivos \\
\hline \multirow{9}{*}{ 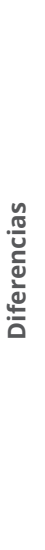 } & Contexto socio-cultural & Bajo & Alto \\
\hline & Acercamiento de familias & Bajo & Alto \\
\hline & $\begin{array}{l}\text { Facilitadores para la } \\
\text { colaboración }\end{array}$ & Docentes & Dirección \\
\hline & Clima organizacional & No favorece & Favorece \\
\hline & Supervisión & No apoya iniciativas & Apoya iniciativas \\
\hline & Definición de colaboración & Centrada en docente & Centrada en estudiante \\
\hline & $\begin{array}{l}\text { Experiencias dentro } \\
\text { (institucionales) }\end{array}$ & Baja cantidad & Gran cantidad \\
\hline & $\begin{array}{l}\text { Experiencias fuera } \\
\text { (pedagógicas) }\end{array}$ & Gran cantidad & Baja cantidad \\
\hline & $\begin{array}{l}\text { Dificultades para la } \\
\text { colaboración }\end{array}$ & Cuestiones personales & Tiempo disponible \\
\hline
\end{tabular}

Fuente: Elaboración propia. 
Existen similitudes que pueden explicar las causas por las que, en estos centros, la colaboración no tuviera mayores impactos. En primer lugar, según los docentes, el uso de la coordinación semanal o el tiempo asignado para la misma eran inadecuados. Este impedimento no era revertido a partir del aprovechamiento de recursos TIC disponibles debido a una actitud neutra por parte de la mayoría de los profesores y las dificultades asociadas a los dispositivos (roturas, falta de reposición, obsolescencia, entre otros).

En segundo lugar, de los testimonios de los informantes se puede inferir que la socialización y reflexión sobre las prácticas es baja a nivel del centro y reducida fuera del centro, mientras que los registros de las experiencias de aula o proyectos compartidos tampoco son socializados entre docentes. Esto contradice la valoración que tienen los docentes sobre los beneficios de la colaboración en tanto la reconocen como un elemento positivo para su práctica profesional pero, en general, no la fomentan.

En tercer lugar, no parece haber una relación entre el estímulo de la supervisión a la colaboración y las experiencias de colaboración y socialización. Sin embargo, es llamativo el hecho de que las dificultades para colaborar se asocien a cuestiones de la personalidad de cada docente y del tiempo disponible, en especial porque se alinean en el discurso de los informantes a los elementos facilitadores de la colaboración para cada caso.

\section{Discusión y conclusiones}

En este artículo se han presentado los resultados preliminares de un estudio de caso múltiple a partir del desarrollo de un protocolo de análisis basado en tres estrategias: el análisis temático, el análisis de caso y el análisis entre casos. Los objetivos buscan determinar si en el contexto de educación primaria y de educación secundaria los docentes colaboran presencial o virtualmente y si lo hacen con colegas con quienes trabajan en el centro o la interacción se da con docentes de otros centros.

En este marco, los hallazgos primarios -sobre los casos 1 y 8-permiten determinar que, aun visualizando la colaboración como una forma de aprendizaje y actualización profesional, las experiencias de colaboración son limitadas en los centros analizados. En este sentido, los resultados preliminares son coincidentes con estudios previos (Little, 1990; Rosenholtz, 1991; Westheimer, 2008) que, además de establecer categorías para el trabajo desarrollado en colaboración o sin ella, marcan la existencia de un ideario de trabajo compartido cuyos límites y formas no están definidos por la academia ni surgen claros en los centros que se presentan en este artículo.

La falta de acuerdos sobre lo que es colaborar o no genera rupturas no solo conceptuales sino entre los propios docentes. En los centros que se analizan los grupos que colaboran para mejorar la práctica y los logros de sus estudiantes ven con recelo a aquellos docentes que prefieren la autonomía; el clima de trabajo se reciente al sentir que las actividades compartidas son posibles por el esfuerzo de unos pocos. Los grupos de trabajo colaborativo no surgen voluntariamente sino que tienden a ser impuestos, y el uso de las TIC para maximizar los beneficios de la colaboración está en una etapa incipiente. Independiente del desempeño, el desarrollo organizacional de estos centros es acotado y puede constatarse un mayor nivel en el caso 8 dada la apertura a la comunidad y hacia otros centros y organizaciones del entorno. Sin embargo, la falta de socialización de las prácticas y registros y las escasas instancias de reflexión disponibles no permiten, en ninguno de los dos casos, un aprovechamiento del conocimiento creado cerrándose así las posibilidades de aprendizaje organizacional por la falta de desenvolvimiento de estrategias de intercambio de conocimientos generados, a partir de la colaboración. A esto se suma la actitud pasiva frente a las TIC y el poco uso que se da a estas herramientas para el desarrollo profesional y la colaboración entre colegas, posiblemente debido a factores organizacionales que no incentivan su uso (Meneses et al., 2012). 
Si bien en este punto no se puede arribar a resultados concluyentes debido que el análisis solo involucra dos casos, es pertinente remarcar las limitaciones de los subsistemas en este tipo de centros donde la coordinación semanal no es aprovechada para el intercambio docente sobre cuestiones pedagógicas. Asimismo, la falta de explotación de los recursos TIC desplegados por el Plan Ceibal de manera universal entre maestros, profesores, directores e inspectores debilita las posibilidades de lograr bases de datos con proyectos y estrategias que movilicen a la reflexión sobre las prácticas. Se reducen, además, los aportes a la mejora de las estrategias didácticas y por tanto al progreso en el desempeño de los estudiantes, y se recarga a los docentes en la planificación de actividades y recursos que podrían compartirse de manera más sistematizada.

Quizá lo antes expuesto constituya una barrera a la innovación y un contrapeso a los cambios necesarios en el sistema educativo actual, comenzando por la sensibilización de los docentes hacia el trabajo compartido, evidenciándose así una necesidad de cambios en el ámbito de la formación del profesorado.

\section{Referencias bibliográficas}

Barfield, A. (2016). Collaboration. ELT Journal, 70(2), 222-224.

Bazeley, P. (2013). Qualitative data analysis. Practical strategies. London: Sage.

Bell, J. (2005). Doing your Research Project. New York: Open University Press.

Castañeda, L., \& Adell, J. (2011). El desarrollo profesional de los docentes en entornos personales de aprendizaje (PLE). En R. Roig Vila \& C. Laneve (Eds.), La práctica educativa en la sociedad de la información. Innovación a través de la investigación. La pratica educativa nella società dell'informazione. L'innovazione attraverso la ricerca (pp. 83-95). Alcoy - Brescia: Marfil \& La Scuola Editrice.

Chmiliar, L. (2010). Multiple-case design. En A. Mills, G. Eurepos \& E. Wiebe (Eds.), Encyclopedia of case study research (pp. 582-584). Thousand Oaks: Sage.

Crano, W., Brewer, M. \& Lac, A. (2015). Principles and methods of social research (3rd ed.). New York: Taylor \& Francis.

Datnow, A. (2011). Collaboration and contrived collegiality: Revisiting Hargreaves in the age of accountability. Journal of Educational Change, 12(2), 147-158.

Drossel, K., Eickelmann, B. \& Gerick, J. (2017). Predictors of teachers' use of ICT in school - the relevance of school characteristics, teachers' attitudes and teacher collaboration. Education and Information Technologies, 22(2), 551-573.

Flyvbjerg, B. (2006). Five misunderstandings about case-study research. Qualitative Inquiry, $12(2), 219-245$.

Fraenkel, J. \& Wallen, N. (2009). How to design and evaluate research in education (7th ed.). New York: McGraw-Hill.

Friend, M. \& Cook, L. (2013). Interactions: collaboration skills for school professionals (7th ed.). Boston: Pearson. 
Fullan, M. \& Hargreaves, A. (1991). What's worth fighting for? Working together for your school. Toronto: Ontario Public School Teachers' Federation.

Gairín, J., Muñoz, J. \& Rodríguez-Gómez, D. (2009). Estadios organizativos y gestión del conocimiento en instituciones educativas. Revista de Ciencias Sociales, 15(4), 620-634.

Gairín, J. \& Rodríguez-Gómez, D. (2013). Escuelas nodo, aprendizaje distribuido y trabajo colaborativo del profesorado. En J. García \& G. Rabajoli (Eds.), Aprendizaje abierto y aprendizaje flexible. Más allá de formatos y espacios tradicionales (pp. 19-41). Montevideo: ANEP-Plan Ceibal.

Kelchtermans, G. (2006). Teacher collaboration and collegiality as workplace conditions. A review. Zeitschrift für Pädagogik, 52(2), 220-237.

Kyndt, E., Gijbels, D., Grosemans, I. \& Donche, V. (2016). Teachers' everyday professional development: Mapping informal learning activities, antecedents, and learning outcomes. Review of Educational Research, 86(4), 1111-1150.

Lavié, J. (2009). El trabajo colaborativo del profesorado. Un análisis crítico de la cultura organizativa. Sevilla: Comunicación Social.

Little, J. (1990). The persistence of privacy: autonomy and initiative in teachers' professional relations. Teachers College Record, 91(4), 509-536.

Meneses, J., Fàbregues, S., Rodríguez-Gómez, D. \& Ion, G. (2012). Internet in teachers' professional practice outside the classroom: examining supportive and management uses in primary and secondary schools. Computers \& Education, 59(3), 915-924.

Miles, M., \& Huberman, M. (1994). Qualitative Data Analysis: An expanded sourcebook (2nd ed.). Thousand Oaks: Sage.

Ostovar-Nameghi, S. \& Sheikhahmadi, M. (2016). From teacher isolation to teacher collaboration: Theoretical perspectives and empirical findings. English Language Teaching, 9(5), 197-205.

Patton, M. Q. (2002). Qualitative research \& evaluation methods. Thousand Oaks: Sage.

Ronfeldt, M. (2016). Improving teaching through collaboration. Recuperado 27 de julio de 2016, a partir de http://www.shankerinstitute.org/blog/Ronfeldt

Ronfeldt, M., Owens, S., McQueen, K. \& Grissom, J. (2015). Teacher collaboration in instructional teams and student achievement. American Educational Research Journal, 52(3), 475-514.

Rosenholtz, S. (1985). Effective schools: Interpreting the evidence. American Journal of Education, 93(3), 352.

Rosenholtz, S. (1991). Teachers' workplace: The social organization of schools. New York: Teachers College Press. 
Sabariego, M. \& Bisquerra, R. (2009). Fundamentos metodológicos de la investigación educativa. En R. Bisquerra (Ed.), Metodología de la investigación (2.a ed., pp. 19-49). Madrid: La Muralla.

Saldaña, J. (2009). The Coding Manual for qualitative researchers. London: Sage.

Stake, R. (1995). The art of case study research. Thousand Oaks: Sage.

Stake, R. (1999). Investigación con estudio de casos (2.a ed.). Madrid: Morata.

Stake, R. (2006). Multiple case study analysis. New York: The Guilford Press.

Suárez, M., Almerich, G., Díaz, I. \& Fernández, R. (2012). Competencias del profesorado en las TIC. Influencia de factores personales y contextuales. Universitas Psychologica, 11(1), 293-309.

Vaillant, D. (2010). Políticas para un desarrollo profesional docente efectivo. En C. Vélaz de Medrano \& D. Vaillant (Eds.), Aprendizaje y desarrollo profesional docente (pp. 29-37). Madrid: OEl-Fundación Santillana.

Vangrieken, K., Dochy, F., Raes, E. \& Kyndt, E. (2015). Teacher collaboration: a systematic review. Educational Research Review, 15, 17-40.

Westheimer, J. (1999). Communities and consequences: an inquiry into ideology and practice in teachers' professional work. Educational Administration Quarterly, 35(1), 71-105.

Westheimer, J. (2008). Learning among colleagues: teacher community and the shared enterprise of education. En M. Cochran-Smith, D. Feiman-Nemser, J. Mclntyre, \& K. Demers (Eds.), Handbook of research in teacher education (pp. 756-785). New York: Routledge.

Yin, R. (2014). Case study research: design and methods (5th ed.). Los Ángeles: Sage. 